Fountain Journal of Natural and Applied Sciences: 2016; 5(2): 7 - 11

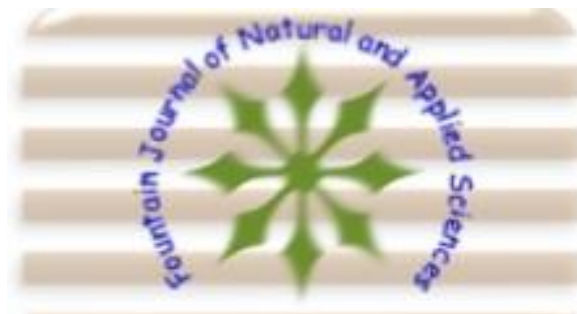

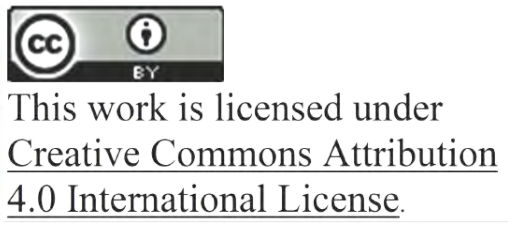

DOI: 10.53704/fujnas.v5i2.101

A publication of College of Natural and Applied Sciences, Fountain University, Osogbo, Nigeria.

Journal homepage: www. fountainjournals.com

ISSN: 2354-337X (Online), 2350-1863 (Print)

\title{
Fuzzy Bigroup from another Viewpoint
}

\footnotetext{
${ }^{1}$ Akinola, L. S., ${ }^{2}$ Oyebo, Y. T., ${ }^{1}$ Abolarin, E. O., ${ }^{1}$ Udoye, A. M., ${ }^{1}$ Salaudeen, L. $O$.

1 Department of Mathematics, Faculty of Science, Federal University, Oye-Ekiti, Ekiti State, Nigeria.

2 Department of Mathematics, Lagos State University, Ojoo-Lagos.lagos State, Nigeria.
}

\begin{abstract}
In group theory, given two groups $G$ and $H$, it is possible to construct a new group from the Cartesian product of $G$ and $H, G \times H$. With this as a motivation, we replicate this concept in fuzzy group algebra. In this paper, we take a slight deviation from the familiar definition of fuzzy bigroup by looking at fuzzy bigroup from the idea of Cartesian product of groups. We define Cartesian fuzzy function on groups and give examples. We also define Cartesian fuzzy bigroup and study some of its basic properties.
\end{abstract}

Keywords: Bigroups, Cartesian fuzzy function, Fuzzy bigroups.

\section{Introduction}

Zadeh (1965) introduced fuzzy sets. Since then, this idea has been extended to many branches of mathematics. Fuzzy subsystems of

algebraic structures have been introduced and extensively studied by several mathematicians. In particular, in the areas of fuzzy group theory, Rosenfeld (1971) introduced the notion of fuzzy subgroups. He gave the conditions for a fuzzy subsets of a non-empty set to be a fuzzy subgroup and discussed its characterization.

Maggu (1994) was the first to introduce the notion of bigroup. He defined a bigroup and investigated whether or not those properties satisfied by groups are also satisfied by bigroups. These notions were further studied by (Vasantha and Meiyappan, 2003). These authors gave modifications of some results earlier proved by Maggu. Among these results were the characterization theorems for sub-bigroup. Meiyappan et al. (1998) introduced the notion of fuzzy union of any two fuzzy subsets of two distinct sets. They used this idea to define fuzzy sub-bigroup of a bigroup and studied its properties. They further established the relationship between fuzzy subgroup of a group and fuzzy sub-bigroup of a bigroup.

Akinola and Agboola (2012) pointed out the lapses observed in the fuzzy bigroup defined by Meiyappan et al. (1998). According to Meiyappan, if $G=\left(G_{1} \cup G_{2},+, \circ\right)$ is a bigroup, then $\mathrm{\gamma}: G \rightarrow[0,1]$ is said to be a fuzzy sub-bigroup of the bigroup $G$ if there exist two fuzzy subsets $V_{1}$ (of $G_{1}$ ) and $Y$ ${ }_{2}$ (of $G_{2}$ ) such that: $\left(Y_{1},+\right)$ is a fuzzy subgroup of $\left(G_{1},+\right),\left(\gamma_{2}, i\right)$ is a fuzzy subgroup of $\left.G_{2}, i\right)$, and $y=$ $\mathrm{V}_{1} \cup \mathrm{V}_{2}$. Even though, this definition has a clear meaning from the context, it is observed that this can still be improved upon especially with the notation. $\left(\mathrm{Y}_{1},+\right)$, and $\left(\mathrm{Y}_{2}, i\right)$ in the definition look as if " + " and "."are operations defined on the fuzzy

*Corresponding author: +2348038946313

Email address: lukman.akinola@fuoye.edu.ng 
Fountain Journal of Natural and Applied Sciences: 2016; 5(2): 7 - 11

sets $\gamma_{1}$ and $\gamma_{2}$ respectively. In this paper, we take a slight deviation from the familiar definition of fuzzy bigroup by looking at fuzzy bigroup from the idea of Cartesian product of sets (groups). We define Cartesian fuzzy function on groups and give examples. We also give conditions to be satisfied for Cartesian fuzzy function to be a fuzzy bigroup and study some of its fundamental properties.

\section{Preliminaries}

We present in this section some of the results we found useful in establishing our results in the next section. For details, please see the references.

Note that fuzzy subgroup and fuzzy group are used interchangeably likewise fuzzy sub bigroup and fuzzy bigroup just like fuzzy set and fuzzy subset.

Definition (2.1): A set $(G,+,)^{\circ}$ with two binary operations " + " and "." is called a bi-group if there exist two proper subsets $G_{1}$ and $G_{2}$ of $G$ such that

(i) $G=G_{1} \cup G_{2}$,

(ii) $\left(G_{1},+\right)$ is a group,

(iii) $\left(G_{2}, \cdot\right)$ is a group.

Definition (2.2): A subset $H(\neq \phi)$ of a bi-group $(G,+$,$) is called a sub bi-group of G$ if $H$ itself is a bi-group under the operations of " + " and "." defined on $G$.

Theorem (2.3): Let $(G,+$,$) be a bi-group. If the$ subset $H(\neq \phi)$ of a bi-group $G$ is a sub bi-group of $G$, then $(H,+)$ and $(H, \cdot)$ are generally not groups.

Definition (2.4): Let $G$ be a non empty set. $A$ mapping $\gamma: G \rightarrow[0,1]$ is called a fuzzy subset of $G$.

Definition (2.5): Let $\gamma$ be a fuzzy set in a set $G$. Then, the $t$-level subset of $\gamma$, denoted by $\gamma_{t}$ is defined as: $\gamma_{t}=\{x \in G: \gamma(x) \geq t\}$ for $t \in[0,1]$.

Definition (2.6): Let $\gamma$ be a fuzzy set in a group $G$. Then, $\gamma$ is said to be a fuzzy subgroup of $G$, if the following hold:

(i) $\quad \gamma(x y) \geq \min \{\gamma(x), \gamma(y)\} \forall x, y \in G$;

(ii) $\quad \gamma\left(x^{-1}\right)=\gamma(x) \forall x \in G$.

Proposition (2.7): Let $G$ be a group with the unit element $e$ and $\gamma$ be a fuzzy subgroup of $G$. For all $x, y \in G$,
(i) $\quad \gamma(x) \leq \gamma(e)$,
(ii) $\quad \gamma(x)=\gamma\left(x^{-1}\right)$,
(iii) $\quad \gamma\left(x y^{-1}\right)=\gamma(e) \Rightarrow \gamma(x)=\gamma(y)$.

Definition (2.8): Let $\gamma$ be a fuzzy subgroup of $G$. Then, the level subset $\gamma_{t}$, for $t \in \operatorname{Im} \gamma$ is a subgroup of $G$ and is called the level subgroup of $G$.

Definition (2.9): Let $\gamma_{1}$ be a fuzzy subset of a set $X_{1}$ and $\gamma_{2}$ be a fuzzy subset of a set $X_{2}$, then the fuzzy union of the sets $\gamma_{1}$ and $\gamma_{2}$ is defined as a function $\gamma_{1} \cup \gamma_{2}: X_{1} \cup X_{2} \rightarrow[0,1]$ given by:

$\left(\gamma_{1} \cup \gamma_{2}\right)(x)=\left\{\begin{array}{c}\max \left(\gamma_{1}(x), \gamma_{2}(x)\right) \text { if } x \in X_{1} \cap X_{2}, \\ \gamma_{1}(x) \text { if } x \in X_{1} \text { and } x \notin X_{2}, \\ \gamma_{2}(x) \text { if } x \in X_{2} \text { and } x \notin X_{1} .\end{array}\right.$

Definition (2.10): Let $G=\left(G_{1} \cup G_{2},+, ;\right)$ be a bigroup. Then $\mu: G \rightarrow[0,1]$ is said to be a fuzzy subbigroup of the bigroup $G$ if there exist two fuzzy subsets $\mu_{1}$ (of $G_{1}$ ) and $\mu_{2}$ (of $G_{2}$ ) such that:

(i) $\left(\mu_{1},+\right)$ is a fuzzy subgroup of $\left(G_{1},+\right)$,

(ii) $\left(\mu_{2},\right)$ is a fuzzy subgroup of $\left(G_{2},{ }^{\prime}\right)$, and

(iii) $\quad \mu=\mu_{1} \cup \mu_{2}$.

\section{The Results}

The notations used in this section are conventional. $\times$ represents both the usual product of real numbers and Cartesian product of sets. In each case, the meaning is always clear from the context. $\Lambda$ stands for minimum as widely used in lattice theory. Let $(G, *)$ and $(H, \star)$ be groups. Let $\phi, \psi: G, H \rightarrow(0,1)$ be fuzzy subgroups of $G$ and $H$ respectively. For any element $g_{i} \in G$, let $\phi\left(g_{i}\right)$ be a membership grade assigned to $g_{i} \in G$ in $(0,1)$. Similarly $\psi\left(h_{j}\right)$ denotes the membership grade of element $h_{j} \in H$ in $(0,1)$.

Definition (3.1): Let $\eta: G \times H \rightarrow(0,1)$ be defined by $\eta(g, h)=\phi(g) \times \psi(h)$ for $g \in G$ and $h \in H, \eta$ is called a Cartesian fuzzy function of the fuzzy groups $\phi$ and $\psi$.

$\eta(h, g): H \times G \rightarrow(0,1)$ is defined in a similar way.

Proposition (3.2): For any groups $G$ and $H$, $\eta(g, h)=\eta(h, g)$.

Proof: This is obvious since $\phi(g) \times \psi(h)=\psi(h) \times \phi(g)$ for $g \in G$ and $h \in H$ where $\phi(g), \psi(h) \in(0,1)$.

Example (3.3): Let $(G, *)$ and $(H, \circ)$ be groups as shown in the following Cayley table; 
Fountain Journal of Natural and Applied Sciences: 2016; 5(2): 7 - 11

\section{Cayley tables of $(G, *)$ and $(H \circ)$}

\begin{tabular}{|l|l|l|l|l|l|l|}
\hline$G$ & $i$ & $I$ & $m$ & $n$ & 0 & $p$ \\
\hline$I$ & $i$ & $I$ & $m$ & $n$ & $o$ & $p$ \\
\hline$I$ & $I$ & $m$ & $i$ & $p$ & $n$ & 0 \\
\hline$m$ & $m$ & $i$ & $I$ & $o$ & $p$ & $n$ \\
\hline$n$ & $n$ & $o$ & $P$ & $i$ & $l$ & $m$ \\
\hline 0 & 0 & $p$ & $n$ & $m$ & $i$ & $l$ \\
\hline
\end{tabular}

Define a fuzzy set $\phi: G \rightarrow(0,1)$ by

$$
\phi(x)=\left\{\begin{array}{c}
0.8 \text { if } x=i, \\
0.6 \text { if } x \in\{o, p\}, \\
0.5 \text { if } x \in\{l, m, n\}
\end{array}\right.
$$

and another fuzzy set $\psi: H \rightarrow(0,1)$ by:

$\psi(e)=1, \quad \psi(a)=0.5, \quad \psi(c)=0.3$.

It is easy to see that both $\phi$ and $\psi$ are fuzzy subgroups of groups $G$ and $H$ respectively.

The Cartesian products of the groups $G$ and $H$ is given as:

$G \times H=\{(\mathrm{i}, \mathrm{e}), \quad(\mathrm{i}, \mathrm{a}), \quad(\mathrm{i}, \mathrm{b}),(\mathrm{i}, \mathrm{c}), \quad(1, \mathrm{e}), \quad(1, \mathrm{a})$,

$(1, b), \quad(1, c), \quad(m, e), \quad(m, a), \quad(m, b), \quad(m, c)\}$

By definition (3.1), $\eta: G \times H \rightarrow(0,1)$ are given as;

$\{0.80,0.40,0.24,0.24,0.5,0.25,0.15,0.15$,

$0.5,0.25,0.15,0.15,0.15,0.25$,

$0.15,0.15,0.42,0.30,0.18,0.18,0.6,0.30,0.24,0.24\}$

Proposition (3.4): For any groups $G$ and $H$ , $\eta\left(e_{1}, e_{2}\right) \geq \eta(g, h), \quad g \in G$ and $h \in H$ where $e_{1}$ is the identity element of $G$ and $e_{2}$ is the identity element of $H$.

Proof: Let $\phi: G \rightarrow(0,1)$ and $\psi: G \rightarrow(0,1)$ be fuzzy subgroups of groups $G$ and $H$ respectively. $\eta\left(e_{1}, e_{2}\right)=\phi\left(e_{1}\right) \times \psi\left(e_{2}\right)$. By proposition (2.7), for any $g \in G, \phi\left(e_{G}\right) \geq \phi(g)$ and for any $h \in H$, $\psi\left(e_{H}\right) \geq \phi(h)$. Therefore, for $g, h \in G \times H, \phi\left(e_{1}\right) \times \psi\left(e_{2}\right) \geq \phi(g) \times \psi(h)$.

Hence $\eta\left(e_{1}, e_{2}\right) \geq \eta(g, h)$

Definition (3.5): The Cartesian fuzzy function $\eta: G \times H \rightarrow(0,1)$ is said to be a fuzzy bigroup if
1. $\eta(g, h) \geq \Lambda\left\{\varnothing\left(g_{1}\right), \varnothing\left(g_{2}\right)\right\} \times$

$\Lambda\left\{\psi\left(h_{1}\right), \psi\left(h_{2}\right)\right\}$ for every $g=g_{1} g_{2}, h=h_{1} h_{2}$ in $G$ and $H$.

2. $\eta(g, h) \geq \eta\left(g^{-1}, h^{-1}\right)$.

In sequel, we denote the element $(x, y)$ of the cartesian product of $X$ and $Y$ by $x y$.

\begin{tabular}{|l|l|l|l|l|}
\hline$o$ & $e$ & $a$ & $b$ & $c$ \\
\hline$e$ & $e$ & $a$ & $b$ & $c$ \\
\hline$a$ & $a$ & $e$ & $c$ & $b$ \\
\hline$b$ & $b$ & $c$ & $e$ & $a$ \\
\hline$c$ & $c$ & $b$ & $a$ & $e$ \\
\hline
\end{tabular}

Example (3.6): Let $\phi$ and $\psi: G, H \rightarrow(0,1)$ be fuzzy subgroups of $(G, *)$ and $(H, \star)$ respectively as defined in example(3.3). From the same example, $\eta(m, b)=0.5$.

From the table,

$$
\begin{gathered}
\Lambda\{\varnothing(i), \varnothing(m)\} \times \Lambda\{\psi(e), \psi(l)\} \\
=\Lambda\{\varnothing(i), \varnothing(m)\} \times \Lambda\{\psi(a), \psi(e) \\
=\Lambda\{\varnothing(i), \varnothing(m)\} \times \Lambda\{\psi(b), \psi(e)\} \\
=\Lambda\{\varnothing(l), \varnothing(l)\} \times \Lambda\{\psi(e), \psi(b)\} \\
=\Lambda\{\varnothing(l), \varnothing(l)\} \times \Lambda\{\psi(a), \psi(c)\} \\
=\Lambda\{\varnothing(l), \varnothing(l)\} \times \Lambda\{\psi(b), \psi(e)\} \\
=\Lambda\{\varnothing(m), \varnothing(i)\} \times \Lambda\{\psi(e), \psi(b)\} \\
=\Lambda\{\varnothing(m), \varnothing(i)\} \times \Lambda\{\psi(a), \psi(c)\} \\
=\Lambda\{\varnothing(m), \varnothing(i)\} \times \Lambda\{\psi(b), \psi(e)\} \\
=\Lambda\{\varnothing(m), \varnothing(i)\} \times \Lambda\{\psi(c), \psi(a)\} \\
=\Lambda\{\varnothing(n), \varnothing(p)\} \times \Lambda\{\psi(e), \psi(b)\} \\
=\Lambda\{\varnothing(n), \varnothing(p)\} \times \Lambda\{\psi(a), \psi(c)\} \\
=\Lambda\{\varnothing(n), \varnothing(p)\} \times \Lambda\{\psi(b), \psi(c)\} \\
=\Lambda\{\varnothing(o), \varnothing(n)\} \times \Lambda\{\psi(c), \psi(b)\} \\
=\Lambda\{\varnothing(o), \varnothing(n)\} \times \Lambda\{\psi(a), \psi(c)\} \\
=\Lambda\{\varnothing(o), \varnothing(n)\} \times \Lambda\{\psi(b), \psi(c)\} \\
=\Lambda\{\varnothing(o), \varnothing(n)\} \times \Lambda\{\psi(e), \psi(b)\} \\
=\Lambda\{\varnothing(p), \varnothing(o)\} \times \Lambda\{\psi(b), \psi(c)\} \\
=\Lambda\{\varnothing(o), \varnothing(n)\} \times \Lambda\{\psi(c), \psi(b)\} \\
=\Lambda\{\varnothing(o), \varnothing(n)\} \times \Lambda\{\psi(a), \psi(c)\} \\
=\Lambda\{\varnothing(o), \varnothing(n)\} \times \Lambda\{\psi(b), \psi(c)\} \\
=\Lambda\{\varnothing(o), \varnothing(n)\} \times \Lambda\{\psi(c), \psi(a)\} \\
=0.15 .
\end{gathered}
$$

Also,

$$
\begin{aligned}
\Lambda\{\varnothing(i), \emptyset(m)\} \times \Lambda & \{\psi(c), \psi(a)\} \\
& =\Lambda\{\varnothing(l), \emptyset(l)\} \times \Lambda\{\psi(e), \psi(a)\}
\end{aligned}
$$

$=\Lambda\{\varnothing(n), \varnothing(p)\} \times \Lambda\{\psi(c), \psi(a)\}=\Lambda\{\varnothing(p), \varnothing(o)\} \times$ $\Lambda\{\psi(e), \psi(b)\}=0.25$,

and,

$$
\begin{aligned}
\Lambda\{\varnothing(p), \varnothing(o)\} \times \Lambda & \{\psi(e), \psi(b)\} \\
& =\Lambda\{\varnothing(p), \varnothing(o)\} \times \Lambda\{\psi(a), \psi(c)\} \\
=\Lambda\{\varnothing(p), \varnothing(o)\} & \times \Lambda\{\psi(b), \psi(e)\} \\
& =\Lambda\{\varnothing(p), \varnothing(o)\} \times \Lambda\{\psi(c), \psi(a)\} \\
& =0.18
\end{aligned}
$$




\begin{tabular}{|c|c|c|c|c|c|c|c|c|c|c|c|c|c|c|c|c|c|c|c|c|c|c|c|c|}
\hline$*, 0$ & ie & ía & $\overline{i b}$ & ic & Te & Ta & $\mathrm{Tb}$ & Tc & me & $\mathrm{ma}$ & $\mathrm{Mb}$ & $\mathrm{mc}$ & ne & na & $\mathrm{nb}$ & nc & oe & oa & $a b$ & $O C$ & pe & $\mathrm{pa}$ & $\mathrm{pb}$ & pC \\
\hline ie & ie & ia & $\overline{i b}$ & ic & Te & Ta & $\mathrm{Tb}$ & Tc & $\mathrm{me}$ & $\mathrm{ma}$ & $\mathrm{Mb}$ & $\mathrm{mc}$ & ne & na & $\mathrm{nb}$ & $\mathrm{nc}$ & oe & $0 a$ & $\mathrm{ob}$ & $O C$ & pe & $\mathrm{pa}$ & $\mathrm{pb}$ & $\mathrm{pc}$ \\
\hline ia & ia & ie & ic & ib & Ta & Te & Tc & $7 b$ & $\mathrm{ma}$ & me & Mc & $\mathrm{mb}$ & na & ne & $\mathrm{nc}$ & $\mathrm{nb}$ & oa & oe & OC & $a b$ & pa & pe & $\mathrm{pc}$ & $\mathrm{Pb}$ \\
\hline ib & $\mathrm{ib}$ & ic & ie & ia & $\mathrm{Tb}$ & Tc & Te & Ta & $\mathrm{mb}$ & $\mathrm{mc}$ & $\mathrm{Me}$ & $\mathrm{ma}$ & $\mathrm{nb}$ & $\mathrm{nc}$ & ne & na & $\mathrm{ob}$ & $O C$ & oe & oa & $\mathrm{pb}$ & $\mathrm{pc}$ & pe & $\mathrm{Pa}$ \\
\hline ic & ic & ib & ia & ie & Tc & $\mathrm{Tb}$ & Ta & Te & $\mathrm{mc}$ & $\mathrm{mb}$ & $\mathrm{Ma}$ & $\mathrm{me}$ & $\mathrm{nc}$ & $\mathrm{nb}$ & na & ne & $O C$ & $a b$ & oa & oe & $\mathrm{pC}$ & $\mathrm{pb}$ & pa & $\mathrm{pe}$ \\
\hline Te & Te & Ta & $\mathrm{Tb}$ & Tc & $\mathrm{me}$ & $\mathrm{ma}$ & $\mathrm{mb}$ & $\mathrm{mc}$ & ie & ia & Ib & ic & pe & pa & $\mathrm{pb}$ & $\mathrm{pC}$ & ne & na & $\mathrm{nb}$ & nc & oe & $0 a$ & $\mathrm{ob}$ & $O C$ \\
\hline Ta & Ta & Te & Tc & $\mathrm{Tb}$ & $\mathrm{ma}$ & $\mathrm{me}$ & $\mathrm{mc}$ & $\mathrm{mb}$ & ia & ie & ic & ib & $\mathrm{pa}$ & pe & $\mathrm{pC}$ & $\mathrm{pb}$ & na & ne & $\mathrm{nc}$ & $\mathrm{nb}$ & oa & oe & $O C$ & $\overline{a b}$ \\
\hline $\mathrm{Tb}$ & $\mathrm{Tb}$ & Tc & Te & Ta & $\mathrm{mb}$ & $\mathrm{mc}$ & $\mathrm{me}$ & $\mathrm{ma}$ & ib & ic & ie & ia & $\mathrm{pb}$ & $\mathrm{pc}$ & pe & $\mathrm{pa}$ & $\mathrm{nb}$ & $\mathrm{nc}$ & ne & na & $\mathrm{ob}$ & $O C$ & oe & oa \\
\hline Tc & Tc & $\mathrm{Tb}$ & Ta & Te & $\mathrm{mc}$ & $\mathrm{mb}$ & $\mathrm{ma}$ & $\mathrm{me}$ & ic & ib & ia & ie & $\mathrm{pC}$ & $\mathrm{pb}$ & $\mathrm{pa}$ & pe & $\mathrm{nc}$ & $\mathrm{nb}$ & na & ne & $O C$ & $a b$ & oa & oe \\
\hline $\mathrm{me}$ & $\mathrm{me}$ & $\mathrm{ma}$ & $\mathrm{mb}$ & $\mathrm{mc}$ & ie & ia & $\mathrm{ib}$ & ic & Te & Ta & $\mathrm{Tb}$ & Tc & oe & oa & $\mathrm{ob}$ & $O C$ & pe & $\mathrm{pa}$ & $\mathrm{pb}$ & $\mathrm{pC}$ & ne & na & $\mathrm{nb}$ & $\mathrm{nc}$ \\
\hline $\mathrm{ma}$ & $\mathrm{ma}$ & $\mathrm{me}$ & $\mathrm{mc}$ & $\mathrm{mb}$ & ia & ie & ic & $\mathrm{ib}$ & Ta & Te & Tc & $\mathrm{Tb}$ & oa & oe & $O C$ & $a b$ & $\mathrm{pa}$ & pe & $\mathrm{pC}$ & $\mathrm{pb}$ & na & ne & $\mathrm{nc}$ & $\mathrm{nb}$ \\
\hline $\mathrm{mb}$ & $\mathrm{mb}$ & $\mathrm{mc}$ & $\mathrm{me}$ & $\mathrm{ma}$ & ib & ic & ie & $i a$ & $\mathrm{~Tb}$ & Tc & Te & Ta & $\mathrm{ob}$ & $O C$ & $0 \mathrm{e}$ & $0 a$ & $\mathrm{pb}$ & $\mathrm{pc}$ & pe & $\mathrm{pa}$ & $\mathrm{nb}$ & $\mathrm{nc}$ & ne & na \\
\hline $\mathrm{mc}$ & $\mathrm{mc}$ & $\mathrm{mb}$ & $\mathrm{ma}$ & $\mathrm{me}$ & ic & ib & ia & ie & Tc & $\mathrm{Tb}$ & Ta & Te & $O C$ & $a b$ & oa & oe & $\mathrm{pC}$ & $\mathrm{pb}$ & $\mathrm{pa}$ & pe & $\mathrm{nc}$ & $\mathrm{nb}$ & na & ne \\
\hline ne & ne & na & $\mathrm{nb}$ & $\mathrm{nc}$ & oe & oa & $\mathrm{ob}$ & $O C$ & pe & $\mathrm{pa}$ & $\mathrm{pb}$ & $\mathrm{pC}$ & ie & ia & $i b$ & ic & Te & Ta & $\mathrm{Tb}$ & Tc & $\mathrm{me}$ & $\mathrm{ma}$ & $\mathrm{mb}$ & $\mathrm{mc}$ \\
\hline na & na & ne & $\mathrm{nc}$ & $\mathrm{nb}$ & oa & oe & $O C$ & $\mathrm{ob}$ & $\mathrm{pa}$ & pe & $\mathrm{pC}$ & $\mathrm{pb}$ & ia & ie & ic & $i b$ & la & Te & Tc & $\mathrm{Tb}$ & $\mathrm{ma}$ & $\mathrm{me}$ & $\mathrm{mc}$ & $\mathrm{mb}$ \\
\hline $\mathrm{nb}$ & $\mathrm{nb}$ & $\mathrm{nc}$ & ne & na & $\mathrm{ob}$ & $O C$ & oe & oa & $\mathrm{pb}$ & $\mathrm{pC}$ & pe & $\mathrm{pa}$ & $\mathrm{ib}$ & ic & ie & ia & $\mathrm{Tb}$ & Tc & Te & Ta & $\mathrm{mb}$ & $\mathrm{mc}$ & $\mathrm{me}$ & $\mathrm{ma}$ \\
\hline $\mathrm{nc}$ & $\mathrm{nc}$ & $\mathrm{nb}$ & na & ne & $O C$ & $\mathrm{ob}$ & oa & oe & $\mathrm{pC}$ & $\mathrm{pb}$ & $\mathrm{pa}$ & pe & ic & ib & ia & ie & Tc & $\mathrm{Tb}$ & Ta & Te & $\mathrm{mc}$ & $\mathrm{mb}$ & $\mathrm{ma}$ & $\mathrm{me}$ \\
\hline oe & oe & oa & $\mathrm{ob}$ & $O C$ & pe & $\mathrm{pa}$ & $\mathrm{pb}$ & $\mathrm{pC}$ & ne & na & $\mathrm{nb}$ & $\mathrm{nc}$ & $\mathrm{me}$ & $\mathrm{ma}$ & $\mathrm{mb}$ & $\mathrm{mc}$ & ie & ia & $\mathrm{ib}$ & ic & Te & la & $\mathrm{lb}$ & Tc \\
\hline oa & oa & $0 \mathrm{e}$ & $O C$ & $\mathrm{ob}$ & $\mathrm{pa}$ & pe & $\mathrm{pC}$ & $\mathrm{pb}$ & na & ne & $\mathrm{nc}$ & $\mathrm{nb}$ & $\mathrm{ma}$ & $\mathrm{me}$ & $\mathrm{mc}$ & $\mathrm{mb}$ & ia & ie & ic & ib & Ta & Te & Tc & $\mathrm{Tb}$ \\
\hline $\mathrm{ob}$ & $\mathrm{ob}$ & $O C$ & oe & oa & $\mathrm{pb}$ & $\mathrm{pC}$ & pe & $\mathrm{pa}$ & $\mathrm{nb}$ & $\mathrm{nC}$ & ne & na & $\mathrm{mb}$ & $\mathrm{mc}$ & $\mathrm{me}$ & $\mathrm{ma}$ & $\mathrm{ib}$ & ic & ie & ia & Tb & Tc & Te & Ta \\
\hline$O C$ & $O C$ & $\mathrm{ob}$ & oa & oe & $\mathrm{pC}$ & $\mathrm{pb}$ & $\mathrm{pa}$ & pe & $\mathrm{nc}$ & $\mathrm{nb}$ & na & ne & $\mathrm{mc}$ & $\mathrm{mb}$ & $\mathrm{ma}$ & $\mathrm{me}$ & ic & $\mathrm{ib}$ & ia & ie & Tc & $\mathrm{lb}$ & Ta & Te \\
\hline $\mathrm{pe}$ & pe & $\mathrm{pa}$ & $\mathrm{pb}$ & DC & ne & na & $\mathrm{nb}$ & $\mathrm{nc}$ & oe & oa & $\mathrm{ob}$ & $O C$ & le & Ta & $\mathrm{Tb}$ & Tc & $\mathrm{me}$ & $\mathrm{ma}$ & $\mathrm{mb}$ & $\mathrm{mc}$ & ie & ia & ib & ic \\
\hline pa & $\mathrm{pa}$ & pe & $\mathrm{pC}$ & $\mathrm{pb}$ & na & ne & $\mathrm{nc}$ & $\mathrm{nb}$ & oa & oe & $O C$ & $\mathrm{ob}$ & Ta & Te & Tc & $\mathrm{Tb}$ & $\mathrm{ma}$ & $\mathrm{me}$ & $\mathrm{mc}$ & $\mathrm{mb}$ & ia & ie & ic & ib \\
\hline $\mathrm{pb}$ & $\mathrm{pb}$ & $\mathrm{pC}$ & pe & $\mathrm{pa}$ & $\mathrm{nb}$ & $\mathrm{nc}$ & ne & na & $\mathrm{ob}$ & $O C$ & oe & $0 a$ & $\mathrm{~Tb}$ & Tc & Te & Ta & $\mathrm{mb}$ & $\mathrm{mc}$ & $\mathrm{me}$ & $\mathrm{ma}$ & $\mathrm{ib}$ & ic & ie & ia \\
\hline $\mathrm{pC}$ & $\mathrm{pC}$ & $\mathrm{pb}$ & $\mathrm{pa}$ & pe & $\mathrm{nc}$ & $\mathrm{nb}$ & na & ne & $O C$ & $\mathrm{ob}$ & oa & oe & Tc & $7 \mathrm{~b}$ & Ta & Te & $\mathrm{mc}$ & $\mathrm{mb}$ & $\mathrm{ma}$ & $\mathrm{me}$ & ic & ib & ia & ie \\
\hline
\end{tabular}

\section{It is easy to see that}

$\eta(m, b) \geq \phi\left(m_{1}, m_{2}\right) \times \psi\left(b_{1}, b_{2}\right)$ for all $m=m_{1} m_{2}$ and $b=b_{1} b_{2}$ in $G$ and $H$

respectively, also that $\eta(m, b) \geq \eta\left(m^{-1}, b^{-1}\right)$.

It is a routine to see that

$$
\eta(g, h) \geq \Lambda\left\{\varnothing\left(g_{1}\right), \varnothing\left(g_{2}\right)\right\} \times \Lambda\left\{\psi\left(h_{1}\right), \psi\left(h_{2}\right)\right\}
$$

for every $g=g_{1} g_{2}, h=h_{1} h_{2}$ in $G$ and $H$ and $\eta(g, h) \geq \eta\left(g^{-1}, h^{-1}\right)$.

Hence, $\eta: G \times H \rightarrow(0,1)$ is a fuzzy bigroup of the bigroup $G$ and $H$.

Theorem (3.7): Let $\alpha$ and $\beta$ be fuzzy subgroups of $G$ and $H$ respectively such that $\alpha(g) \leq \phi(g)$ and $\beta(h) \leq \psi(h)$ for every $(g, h) \in G \times H$. Let $\eta: G \times H \rightarrow(0,1)$ be a fuzzy bigroup of $G \times H$ then;

(i) $\rho \leq \eta$ for every $g, h \in G \times H$,

(ii) the fuzzy function $\rho=\alpha \times \beta: G \times H \rightarrow(0,1)$ is also a fuzzy bigroup of the bigroup $G \times H$.

Proof: (i) Since $\alpha(g) \leq \phi(g)$ and $\beta(h) \leq \psi(h)$ for every $(g, h) \in G \times H$, then $\Lambda\left\{\varnothing\left(g_{1}\right), \varnothing\left(g_{2}\right)\right\} \times$ $\Lambda\left\{\psi\left(h_{1}\right), \psi\left(h_{2}\right)\right\} \leq \Lambda\left\{\alpha\left(g_{1}\right), \alpha\left(g_{2}\right)\right\} \times$ $\Lambda\left\{\beta\left(h_{1}\right), \beta\left(h_{2}\right)\right\}$.

Therefore $\alpha \times \beta \leq \varnothing \times \psi(h)$. This implies that $\rho \leq \eta$.

Definition (3.8): Let $\eta: G \times H \rightarrow(0,1)$ be a fuzzy bigroup. The bilevel subset of the fuzzy bigroup $\eta$ denoted by $\eta_{t}$ is defined as

$\eta_{t}:=\{(g, h) \in G \times H: \eta(g, h) \geq t\}$ for $t \in[0,1]$.

The following proposition is a direct consequence of Definition 3.8 and the conventional definition of level subset of a set.

Proposition (3.9): Let $\phi_{t}$ be a $t$ - level subset of the group $G$ and $\psi_{s}$ be a $s$-level subset of the group $H$, then:

(i) $\quad \eta_{t}$ is a $t$-bilevel subset of the bigroup $G \times H$ if $t \leq s$

(ii) $\quad \eta_{s}$ is a $s$-bilevel subset of the bigroup $G \times H$ if $s \leq t$.

Proof: (i) For $x \in G$ such that $\phi(x) \geq t$, then $x \in \phi_{t}$ and for $y \in H$ such that $\psi(y) \geq s$, then $y \in \psi_{s}$.

Therefore,

for

$(x, y) \in \phi_{t} \times \psi_{s} \subseteq G \times H, \eta(x, y) \geq \min \{\phi(x), \psi(s)\}$

$\geq \min \{t, s\}=t$.

Hence, $\eta_{t}$ is a bilevel subset of the bigroup $\times H$. The proof of (ii) is similar.

Theorem (3.10): The $t$ - bilevel subset $\eta_{t} \subseteq \phi_{t} \times \psi_{s}$ of the bigroup $G \times H$ is a $t$-bilevel fuzzy sub bigroup of $G \times H$ if the following conditions are satisfied:

(i) $0 \leq s \leq t \leq 1$

(ii) for $t \in \operatorname{Im} \phi$ and $s \in \operatorname{Im} \psi, \phi_{t}$ and $\psi_{s}$ are fuzzy subgroups of $G$ and $H$ respectively.

(iii) $t \leq \min \left\{\phi\left(e_{1}\right), \psi\left(e_{2}\right)\right\}$ 
Fountain Journal of Natural and Applied Sciences: 2016; 5(2): 7 - 11

where $e_{1}$ and $e_{2}$ are the identity elements of $G$ and $H$ respectively.

\section{Proof:}

$\eta_{t}=\{(x, y) \in G \times H: \eta(x, y) \geq t\}$

i.e. $\eta_{t}=\{(x, y) \in G \times H: \phi \times \psi(x, y) \geq t\}$

i.e. $\eta_{t}=\{(x, y) \in G \times H: \phi(x) \geq t, \psi(y) \geq t\}$

Since $0 \leq s \leq t \leq 1$,

$\eta_{t}=\{(x, y) \in G \times H: \phi(x) \geq t, \psi(y) \geq t\}$

$\Rightarrow \eta_{t}=\{(x, y) \in G \times H: \phi(x) \geq t, \psi(y) \geq s\}$

Let $x_{1}, x_{2} \in \phi_{t}$, and since $\phi_{t}$ is a fuzzy subgroup of $G$, then $\varnothing\left(x_{1} x_{2}{ }^{-1}\right) \geq \Lambda\left\{\varnothing\left(x_{1}\right), \varnothing\left(x_{2}\right)\right\}$. Also, for $y_{1}, y_{2} \in \psi_{s}$, since $\psi_{s}$ is a fuzzy subgroup of $H$, then $\psi\left(y_{1} y_{2}^{-1}\right) \geq \Lambda\left\{\psi\left(y_{1}\right), \psi\left(y_{2}\right)\right\}$.

Therefore,

$$
\begin{gathered}
\eta\left(x_{1} x_{2}^{-1}, y_{1} y_{2}^{-1}\right)=\varnothing\left(x_{1} x_{2}^{-1}\right) \times\left(y_{1} y_{2}^{-1}\right) \\
\geq \Lambda\left\{\varnothing\left(x_{1}\right), \varnothing\left(x_{2}\right)\right\} \times \Lambda\left\{\varnothing\left(y_{1}\right), \varnothing\left(y_{2}\right)\right\} .
\end{gathered}
$$

It is easy to infer that $t \leq \min \left\{\phi\left(e_{1}\right), \psi\left(e_{2}\right)\right\}$ where $e_{1}$ and $e_{2}$ are the identity elements of $G$ and $H$ respectively by proposition (3.4). Hence, the result follows.

\section{Conclusion}

We have been able to define fuzzy bigroup from the idea of Cartesian product of groups and construct example. We also discussed some fundamental properties of this fuzzy bigroup.

\section{References}

Akinola, L.S., \& Agboola, A.A. (2012). Permutable and mutually permutable fuzzy bigroup. Proceedings of Jangjeon Mathematical Society 13(1), 98-109.

Maggu, P.L. (1994). On introduction of bigroup concept with its application in industry. Pure Applied Mathematical Science 39, 171-173.

Meiyappan, D. \& Vasantha, W.B.K. (1998). Fuzzy symmetric subgroups and conjugate fuzzy subgroups of a group. Journal of Fuzzy Mathematics. IFMI, 6, 905-913.

Murthuraj, R., \& Rajinkannan, M. (2010). A study on Anti- fuzzy Sub-Bigroup. International Journal of Computer Application, 21, 3135.

Rosenfeld, A. (1971). Fuzzy groups. Journal of Mathematical Analysis and Applications. 35, 512-517.

Vasantha, W.B.K. (2003). Bialgebraic structures and Smarandache bialgebraic structures. American Research Press, Rehoboth, NM,

Zadeh, L.A. (1965). Fuzzy sets. Information and Control, 8, 338-353. 